\title{
Demo:
}

\section{LiTell: Indoor Localization Using Unmodified Light Fixtures}

\author{
Chi Zhang Xinyu Zhang \\ czhang296@wisc.edu xyzhang@ece.wisc.edu \\ University of Wisconsin-Madison
}

\begin{abstract}
Owing to dense deployment of light fixtures and multipath-free propagation, visible light localization technology holds potential to overcome the reliability issue of radio localization. However, existing visible light localization systems require customized light hardware, which increases deployment cost and hinders near term adoption. We present LiTell, a simple and robust localization scheme that employs unmodified fluorescent lights (FLs) as location landmarks and commodity smartphones as light sensors. LiTell builds on the key observation that each FL has an inherent characteristic frequency, which can serve as a discriminative feature. It incorporates a set of sampling, signal amplification and camera optimization mechanisms, that enable a smartphone to capture the extremely weak and high frequency $(>80 \mathrm{kHz})$ features. We have implemented LiTell as a real-time localization and navigation system on Android. In our experiments, LiTell demonstrates high reliability in discriminating different FLs, and great potential to achieve sub-meter granularity.
\end{abstract}

\section{CCS Concepts}

-Computer systems organization $\rightarrow$ Special purpose systems; -Hardware $\rightarrow$ Signal processing systems; Sensors and actuators; Sensor applications and deployments;

\section{Keywords}

Visible light sensing; Visible light localization; Indoor localization

\section{INTRODUCTION}

Indoor localization technology is bringing huge impacts on human activities. Existing market research predicts that location-based services in retail industry alone will generate 10 billion revenues by 2020 [1]. However, after decades of research, there still lacks an indoor localization solution with desired simplicity and robustness. A recent field test of state-of-the-art localization schemes [2] revealed a common set of problems including high deployment overhead and low reliability. The consensus is that robust meter-level indoor localization remains an open problem. Radio based localization techniques are most extensively studied [3-6], however, the elusive na-

Permission to make digital or hard copies of part or all of this work for personal or classroom use is granted without fee provided that copies are not made or distributed for profit or commercial advantage and that copies bear this notice and the full citation on the first page. Copyrights for third-party components of this work must be honored. For all other uses, contact the owner/author(s).

MobiCom'16 October 03-07, 2016, New York City, NY, USA

(C) 2016 Copyright held by the owner/author(s).

ACM ISBN 978-1-4503-4226-1/16/10.

DOI: http://dx.doi.org/10.1145/2973750.2985612
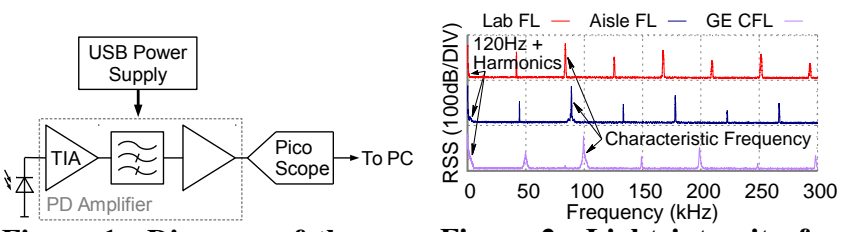

Figure 1: Diagram of the cus- Figure 2: Light intensity fretomized high-speed light sensor. quency components of FLs.

ture of radio signals together with sparse deployment of access points render them less reliable in real environments [2,7]. Meanwhile, visible light (VL) localization techniques hold potential to overcome such fundamental limitations. Using ceiling-mounted LEDs as beaconing devices, VL localization can achieve sub-meter precision [8], and can even determine the orientation of a smartphone [9]. However, almost all existing LED based localization techniques require adding customized beaconing circuits to the LEDs [10], which involves substantial cost and hinders near term adoption [11].

To address these problems, we designed and implemented LiTell, a simple and robust VL localization scheme, which can be immediately used on unmodified light fixtures and commercial-off-the-shelf (COTS) smartphones. LiTell uses fluorescent lights (FLs) as location anchors, and smartphone cameras as receivers. The key hypothesis is that an FL's driver acts as an oscillator with a resonance frequency. Due to unavoidable manufacturing variation, different FLs have different resonance frequencies, which will cause each FL to flicker at a characteristic frequency (CF), a high frequency $(>80 \mathrm{kHz})$ that is not perceptible by human and remains relatively stable in practice. LiTell uses the $\mathrm{CF}$ as a discriminative feature among different FLs which in turn serve as location landmarks. We have tested the hypothesis using a customized high-speed light sensor (Fig. 1). Our experiments demonstrate that the CFs are highly diverse, and are also highly reliable (Sec. 2). LiTell also incorporates image signal processing algorithms that convert the smartphone's camera into a high speed optical sampling device. We have implemented these algorithms on COTS Android phones. Our experiments in a 4-storey office building demonstrate that LiTell can identify the CF features with high reliability under various usage behaviors and environment conditions, and across multiple generations of Android phone models built from 2012 to 2015 . In addition, our field study reveals that LiTell has low and bounded setup cost and unobtrusive fingerprinting process, and is robust against heavy human activities and environment dynamics. To our knowledge, LiTell represents the first ready-to-use, real-time localization system to achieve a combination of such desirable traits.

\section{OVERVIEW}

LiTell employs unmodified fluorescent lights (FLs) as location landmarks. To sparkle the fluorescent tubes, FL's driver circuit 

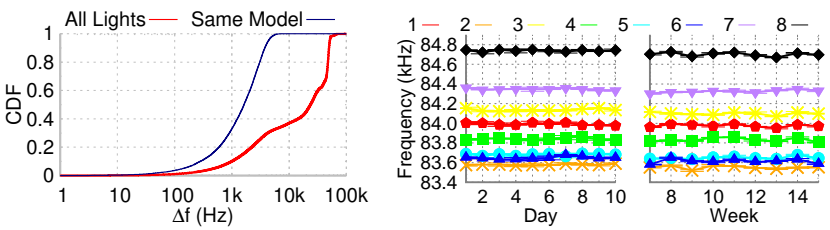

Figure 3: CDF of $\Delta f$ for Figure 4: Short-term and longFLs in the office building.

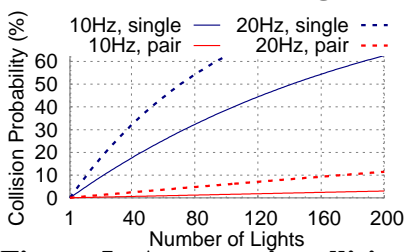

Figure 5: Asymptotic collision probability of single and pair col matching, under different tol- anc erances.

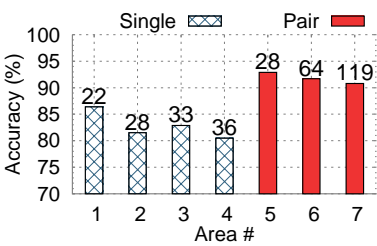

Figure 6: Measured noncollision probability of single and pair matching. Numbers show number of lights in area.

("ballast") converts electricity to a high frequency AC voltage, whose frequency is determined by a few components and reflected in the FL's light emission. Due to manufacturing variation, the value of these components usually varies by $5-20 \%$. As a result, even among FLs of the same model, their frequencies tend to vary.

Fig. 2 plots the frequency domain spectrum of light intensity from 3 example FLs. All the FLs have a fundamental frequency component followed by its harmonics. The dominate frequency occurs at the second harmonic with 20-30dB higher magnitude, which eases detection. Thus, we use the dominate frequency as the FL's characteristic frequency $(C F)$.

Fig. 3 plots the CDF of pairwise differences in CFs $(\Delta f)$ among over $500 \mathrm{FLs}$ in our office building. Only less than $0.1 \%$ of the pairs have $\Delta f \leq 10 \mathrm{~Hz}$, and $0.2 \%$ have $\Delta f \leq 20 \mathrm{~Hz}$, even among FLs of the same model. This confirms the CF as a strong feature to discriminate FLs at a large scale.

To verify the temporal stability of $\mathrm{CF}$, we measure the $\mathrm{CF}$ of 8 randomly selected FLs in our office building. Fig. 4 plot the CF values across days and weeks, which shows reasonable accuracy. However, it also displays some drifts in long term, increasing the risk of feature collision among FLs. As a result, LiTell is designed to tolerate small amount of collisions by using pairs of CFs (of previous and current FL) to match in the database, which significantly lowers the collision probability, as shown by calculation (Fig. 5) and experiments (Fig. 6).

Practical challenges emerge when we try to capture such highfrequency CFs using smartphone cameras, which are designed to capture static or low-rate scenes. To overcome camera's low sampling rate, LiTell exploits the sparsity of the $\mathrm{CF}$ feature and the rolling shutter effect. To combat its low sensitivity, LiTell synthesizes consecutive captures of the FL, isolate the interference from ambient spatial patterns and mitigate the noise from camera hardware. Finally, we use a 2-pass identification process to find the peak in the spectrum that corresponds to the $\mathrm{CF}$.

LiTell can also provide sub-light level localization when finegrained location is needed. The size of each light model can be recorded in the database, which helps LiTell establish a relation between physical size and number of pixels in the captured image. By extracting the light in the image, LiTell can easily recover its relative position and thus user's location with sub-light precision.

\section{DEMONSTRATION}

In this demonstration, we will show the LiTell App and its localization / navigation capabilities. We will also run the prototype fingerprinting database on a laptop, along with the high speed sensor for fingerprinting. This will allow us to demonstrate the server-side $\mathrm{CF}$ extraction algorithms and the fingerprinting process as well. During the demo, we will encourage the audience to carry a smartphone that runs the LiTell App and shows the user's location in real time.

Ideally demonstration of LiTell will require tube FLs, which are available in most places. In case the FLs are not available at the conference site, e.g., due to natural lighting or the use of LEDs, we can bring a few of our own lights. We will bring our own laptops and smartphones, but we do need a desk as well as power outlets. We will setup our own Wi-Fi access point for the smartphones used for demonstration. Our demonstration does not require Internet access. The whole setup process (including fingerprinting the demonstration area) should be less than half an hour.

\section{CONCLUSION}

By peeking into the frequency characteristics of FLs' light emission, we explore the feasibility of using unmodified FL fixtures to build a robust indoor localization system. We present LiTell, a system that can discriminate subtle differences in the weak, high frequency characteristics of FLs' emission, utilizing COTS smartphones' cameras augmented with customized sampling/amplification algorithms. This demo will showcase LiTell in the MobiCom'16 conference site, using unmodified ceiling FL fixtures and commodity smartphones. We believe this will trigger substantial interest and inspire new ideas on low-cost visible light localization.

\section{REFERENCES}

[1] ABI Research, "Indoor Location in Retail: Where Is the Money?" 2013.

[2] D. Lymberopoulos, J. Liu, X. Yang, R. R. Choudhury, V. Handziski, and S. Sen, "A Realistic Evaluation and Comparison of Indoor Location Technologies: Experiences and Lessons Learned," in Proc. of ACM/IEEE IPSN, 2015.

[3] P. Bahl and V. Padmanabhan, "RADAR: an In-Building RF-Based User Location and Tracking System," in Proc. of IEEE INFOCOM, vol. 2, 2000.

[4] Y. Chen, D. Lymberopoulos, J. Liu, and B. Priyantha, "FM-based Indoor Localization," in Proc. of ACM MobiSys, 2012.

[5] K. Chintalapudi, A. Padmanabha Iyer, and V. N. Padmanabhan, "Indoor Localization Without the Pain," in Proc. of ACM MobiCom, 2010.

[6] M. Kotaru, K. Joshi, D. Bharadia, and S. Katti, "SpotFi: Decimeter Level Localization Using WiFi," in Proc. of ACM SIGCOMM, 2015.

[7] H. Liu, Y. Gan, J. Yang, S. Sidhom, Y. Wang, Y. Chen, and F. Ye, "Push the Limit of WiFi Based Localization for Smartphones," in Proc. of ACM MobiCom, 2012.

[8] L. Li, P. Hu, C. Peng, G. Shen, and F. Zhao, "Epsilon: A Visible Light Based Positioning System," in Proc. of USENIX NSDI, 2014.

[9] Y.-S. Kuo, P. Pannuto, K.-J. Hsiao, and P. Dutta, "Luxapose: Indoor Positioning with Mobile Phones and Visible Light," in Proc. of ACM MobiCom, 2014.

[10] J. Armstrong, Y. Sekercioglu, and A. Neild, "Visible Light Positioning: a Roadmap for International Standardization," IEEE Communications Magazine, vol. 51, no. 12, 2013.

[11] U.S. Department of Energy, "Energy Savings Forecast of Solid-State Lighting in General Illumination Applications," Aug. 2014. 